\title{
Does Clinically Important Change in Function After Knee Replacement Guarantee Good Absolute Function? The Multicenter Osteoarthritis Study
}

\author{
Jessica L. Maxwell, David T. Felson, Jingbo Niu, Barton Wise, Michael C. Nevitt, \\ Jasvinder A. Singh, Laura Frey-Law, and Tuhina Neogi
}

ABSTRACT. Objective. Poor functional outcomes post-knee replacement are common, but estimates of its prevalence vary, likely in part because of differences in methods used to assess function. The agreement between improvement in function and absolute good levels of function after knee replacement has not been evaluated. We evaluated the attainment of improvement in function and absolute good function after total knee replacement (TKR) and the agreement between these measures.

Methods. Using data from The Multicenter Osteoarthritis (MOST) Study, we determined the prevalence of achieving a minimal clinically important improvement (MCII, $\geq 14.2 / 68$ point improvement) and Patient Acceptable Symptom State (PASS, $\leq 22 / 68$ post-TKR score) on the Western Ontario and McMaster Universities Osteoarthritis Index (WOMAC) Physical Function subscale at least 6 months after knee replacement. We also assessed the frequency of co-occurrence of the 2 outcomes, and the prevalence according to pre-knee replacement functional status.

Results. We included 228 subjects who had a knee replacement during followup (mean age 65 yrs, mean body mass index $33.4,73 \%$ female). Seventy-one percent attained the PASS for function after knee replacement, while only $44 \%$ attained the MCII. Of the subjects who met the MCII, $93 \%$ also attained the PASS; however, of subjects who did not meet the MCII, 54\% still achieved a PASS. Baseline functional status was associated with attainment of each MCII and PASS.

Conclusion. There was only partial overlap between attainment of a good level of function and actually improving by an acceptable amount. Subjects were more likely to attain an acceptable level of function than to achieve a clinically important amount of improvement post-knee replacement. (First Release Dec 1 2013; J Rheumatol 2014;41:60-4; doi:10.3899/jrheum.130313)

Key Indexing Terms:

KNEE REPLACEMENT

PHYSICAL FUNCTION

OUTCOMES

Post-total knee replacement (TKR) functional limitations such as difficulties with stair-climbing or walking occur in about $10 \%$ to $30 \%$ of patients ${ }^{1,2}$. While this may represent

From the Department of Physical Therapy and Athletic Training, College of Health and Rehabilitation Sciences, Boston University; Clinical Epidemiology Training Unit, Department of Medicine, Boston University School of Medicine, Boston, Massachusetts, USA.

Ms. Maxwell is supported by the American College of Rheumatology REF Research Scientist Development Award and NIDDR H133B100003-11.

The MOST Study is supported by US National Institutes of Health grants: U01-AG18820, U01-AG19069, U01-AG18832, U01-AG18947. This work is also supported by AR-47785.

J.L. Maxwell, DPT, PhD candidate, Clinical Assistant Professor of Physical Therapy; D.T. Felson, MD, MPH, Professor of Medicine; J. Niu, $D S c$, Research Assistant Professor, Boston University; B. Wise, MD, MSc, Assistant Professor of Medicine, University of California Davis; M.C. Nevitt, PhD, MPH, Adjunct Professor of Epidemiology and Biostatistics, University of California, San Francisco; J.A. Singh, MBBS, MPH, Associate Professor of Medicine, University of Alabama, Birmingham; L. Frey-Law, MPT, MS, PhD, Associate Professor of Physical Therapy, University of Iowa; T. Neogi, MD, PhD, Associate Professor of Medicine, Boston University.

Address correspondence to J.L. Maxwell, Boston University, Physical Therapy and Athletic Training, 635 Commonwealth Ave., Boston, Massachusetts 02215,USA.E-mail: jmaxwell@bu.edu

Accepted for publication September 27, 2013. only a minority of persons undergoing TKR, the absolute number affected by functional limitations post-TKR is large given the volume of procedures performed. As the numbers undergoing TKR continue to rise, with an estimated 3.5 million TKR predicted to be performed annually in the United States by 2030, an increase of $>600 \%$ compared with $2005^{3}$, the number of patients with poor functional outcomes will also likely rise. One difficulty in studying the effect of poor functional outcomes post-TKR is that presently no clear consensus exists as to how best to define a successful functional outcome ${ }^{4}$.

Successful functional outcomes post-TKR may be defined by either the degree of improvement or the absolute level, or status, of function post-TKR. Relative improvement from baseline to followup following specific interventions is often reported in terms of a minimal clinically important improvement (MCII). MCII is often determined using an anchor question on improvement, such as "somewhat better"; with MCII thresholds defined by how much improvement on a scale corresponds to "somewhat better" in a certain percentage of patients.

On the other hand, the absolute level, or current status, of

Personal non-commercial use only. The Journal of Rheumatology Copyright @ 
function may be defined using a Patient Acceptable Symptom State (PASS). The PASS is determined using an anchor question about satisfaction with current symptoms or performance ${ }^{5}$, then determining the score at which a particular percentage of subjects report meeting that benchmark. MCII and PASS values are specific to the outcome measurement tool under study and any subscales existing within the tool. The 2 systems also have different values depending on the patient population and intervention received even when using the same outcome measure [e.g., MCII values for knee osteoarthritis (OA) and knee replacement will differ $]^{5,6}$. There is recent evidence that MCII and PASS thresholds for pain after nonsteroidal drug use may be similar to those of several rheumatic diseases ${ }^{7}$; however, it is possible that these may differ from those for a major intervention such as surgery.

It is currently unknown whether achievement of a particular absolute level of function post-TKR is indicative of a minimal clinically important improvement, or how frequently achieving a meaningful relative improvement in function translates into an acceptable level of absolute function after surgery. While a recent study determined MCII and PASS values for persons after TKR in different patient populations ${ }^{8}$, to our knowledge there have not been studies comparing these 2 measurements in a single clinical population. Understanding the relationship between the 2 constructs will provide guidance for future research regarding the optimal definition of good functional outcomes in a TKR population.

The goals of our study were (1) to determine the prevalence of an MCII in function, and an acceptable level of absolute function post-TKR, and (2) to determine the overlap between the two and examine the prevalence of these outcomes according to pre-TKR (baseline) function in a large multicenter cohort study.

\section{MATERIALS AND METHODS}

Study sample. The Multicenter Osteoarthritis (MOST) study is a U.S. National Institutes of Health-funded longitudinal prospective cohort study of 3026 people with or at high risk of knee OA and aged 50 to 79 years at baseline, recruited from Birmingham, Alabama, and Iowa City, Iowa. Study design and subject eligibility have been described elsewhere 9 . The MOST study was approved by the institutional review boards at the University of Iowa; the University of Alabama, Birmingham; the University of California, San Francisco; and Boston University Medical Center.

For our study, we included subjects who had at least 1 TKR since study enrollment with a recorded date of surgery, who also had physical function data obtained at the study visit prior to TKR and a study visit at least 6 months following TKR, to allow for sufficient surgical recovery time ${ }^{10}$. Less than $3 \%$ of subjects had unicompartmental knee replacement and the rest had TKR; thus we use the term TKR to describe our sample. Data collected after revision surgeries were not included. Subjects with exposure and outcome data at each of the assessment time points were included in the analyses, irrespective of whether they developed conditions that could alter function.

Assessment of physical function. Subjects completed the self-report Western Ontario and McMaster Universities Osteoarthritis Index Physical
Function ${ }^{11}$ subscale (WOMAC-PF) at baseline, 30-month, and 60-month study visits.

Assessment of TKR status. Knee replacements were self-reported at MOST clinic visits at baseline, 30 months, or 60 months, or at a telephone interview at 15 months, with $>95 \%$ confirmed by medical records and/or study knee radiographs obtained postsurgery.

Statistical methods. We used 2 measures of TKR functional outcome in this study both calculated from the WOMAC-PF: the MCII and the PASS.

We defined the minimal clinically meaningful amount of improvement from pre-TKR to post-TKR as an improvement of $\geq 14.2$ out of 68 points. This value was determined by transforming the published MCII for function after knee replacement of $20.84 / 100^{6}$ to the raw scale of a total of 68 points for the WOMAC-PF subscale. The original authors established this threshold using the mean score of the subjects who reported feeling "somewhat better" . We defined a level of "acceptable" absolute function post-TKR as a score of $\leq 22 / 68$ using the PASS for post-TKR function ${ }^{12}$. This threshold was determined using the 75th percentile score of all subjects reporting that their function was acceptable. We similarly transformed this from its published value of 67.7/100 (high scores meaning better function) to a score out of 68 with high scores meaning worse function in accordance with the original WOMAC. While the thresholds were established using different percentages of subjects attaining each definition of good function, they are to date the only published MCII and PASS values for function following knee replacement.

We determined the proportion of subjects attaining the MCII and the PASS. We stratified these results by quartiles of pre-TKR function given its influence on post-TKR function ${ }^{13,14}$ and assessed between-group differences using a chi-squared test. We assessed the frequency of attaining the PASS for post-TKR function according to attainment of the MCII. We excluded subjects that had a pre-TKR functional score of $\leq 14$ out of 68 from all analyses, because they were unable to attain the MCII (i.e., their pre-TKR function precluded sufficient improvement to achieve the MCII). We did not differentiate between subjects with 1 or 2 knees replaced in these analyses, or between those with both knees replaced concurrently or staged. We conducted 2 sensitivity analyses. Because of concerns that some pre-TKR subjects had their clinical visit far before their surgery and therefore deterioration in function prior to surgery may not have been recorded, we restricted our analyses of the proportion attaining the MCII to those who had their pre-TKR data assessed within 6 months of their TKR. We also conducted ad hoc analyses of the number of subjects who attained MCII after excluding those subjects who were at the PASS level pre-TKR. Statistical analyses were completed using SAS 9.1 (SAS Software).

\section{RESULTS}

There were 271 subjects with a TKR since baseline. We excluded 38 subjects (14\%) with function scores too low to allow attainment of the MCII, and 5 subjects whose post-TKR study visit was within 6 months of their surgery, leaving 228 subjects in our study sample. Of these, 73 subjects had replacements in both knees (sequentially or concurrently). The majority of subjects were white and female, with a mean body mass index (BMI) in the obese range, and mean WOMAC-PF scores demonstrating moderate functional limitation (Table 1). Compared to a recent study using 2617 of the 3026 MOST participants ${ }^{15}$, the subjects in our study were older, had a higher mean BMI, and there was a higher percentage of women. The average time between the pre-TKR function assessment and date of TKR was 15.5 months (1.3 yrs); the average time between the date of TKR and first postreplacement function

Personal non-commercial use only. The Journal of Rheumatology Copyright $\subset$ 2014. All rights reserved. 
Table 1. Demographic and pre-total knee replacement (TKR) clinical information of the study subjects.

\begin{tabular}{lc}
\hline No. subjects & 228 \\
Mean age at TKR, yrs (SD) & $67(8)$ \\
$\%$ Female & 73 \\
$\%$ White & 85 \\
Pre-TKR BMI, kg/m², mean (SD) & $33(7)$ \\
Pre-TKR WOMAC-PF, mean (range) & $30(15-63)$ \\
Time since pre-TKR visit to TKR, & $16(10)$ \\
$\quad$ mos, mean (SD) & $15(9)$ \\
Time since TKR to outcome assessment, mos, & \\
$\quad$ mean (SD) & 26 \\
Percent at or above PASS for TKR function (pre-TKR) & \\
\hline
\end{tabular}

BMI: body mass index; WOMAC-PF: Western Ontario and McMaster Universities Osteoarthritis Index Physical Function subscale; PASS: Patient Acceptable Symptom State.

assessment was 21.6 months (1.8 yrs; any visit falling within 6 months of surgery was excluded).

Forty-four percent of subjects achieved the MCII after TKR, and seventy-one percent of subjects attained the PASS for post-TKR function (Table 2). Forty-one percent of subjects met both the MCII and PASS. Twenty-six percent met neither outcome criterion. Only 3\% met the MCII but did not meet the PASS, while $30 \%$ did not meet the MCII, but did meet the PASS. Twenty-six percent of subjects had baseline (pre-TKR) function scores at or better than the PASS for TKR function. When the analysis was repeated after excluding these subjects, the proportion that attained the MCII only rose by $5 \%$ to $49 \%$. Of the subjects who met the MCII, 93\% of them also attained the PASS for post-TKR function. However, of the subjects who did not meet the MCII, 54\% of them still achieved the PASS for post-TKR function.

Those with worse baseline function were more likely to attain an MCII ( $\mathrm{p}=0.0006)$, but less likely to attain the PASS for post-TKR function ( $\mathrm{p}<0.0001$; Figure 1). Subjects with the best baseline function were least likely to attain an MCII (28\%), but more likely to attain the PASS for post-TKR function (90\%). Subjects having their TKR more closely following the pre-TKR visit had slightly worse pre-TKR function than those having the TKR more months after the visit $(p=0.02)$. When the analysis was restricted to those who had their pre-TKR assessment within 6 months of their surgery $(n=54)$, the proportion of subjects that attained the MCII rose to $63 \%$. The number of months between pre-TKR visit and TKR was not associated with PASS attainment (OR 0.98, 95\% CI 0.95-1.0), and was only weakly associated with MCII attainment $(0.93,95 \%$ CI 0.90-0.96).

\section{DISCUSSION}

Our study demonstrates that the proportion achieving an MCII after TKR is not the same as the proportion that achieved an acceptable absolute level of function. Although almost all subjects who attained an MCII also reached the threshold for good absolute function (PASS), many subjects who did not achieve an MCII also had good absolute function post-TKR.

The low proportion of subjects with minimal clinically important improvement in function post-TKR is in contrast with another study, in which $64 \%$ of subjects attained the MCII at 2 years post-TKR ${ }^{16}$. However, when we limited the analysis to those with a pre-TKR assessment of function within 6 months of the surgery, 63\% attained the MCII, consistent with these prior estimates. The overall proportion achieving the PASS in our study $(71 \%)$ was consistent with the reported prevalence with self-reported good function post-TKR ${ }^{2,17}$. Of particular interest is that only $41 \%$ of subjects had both a minimal clinically important improvement in function (MCII) and attained a state of acceptable physical function (PASS). Conversely, 26\% of subjects did not attain either benchmark (i.e., neither attained an acceptable level of function, nor an MCII in function), supporting other findings regarding the proportion of patients who continue to report functional limitations after $\mathrm{TKR}^{2}$.

The effect of pre-TKR, or baseline, function on the prevalence of functional outcomes differed for each outcome measure. Subjects with worse pre-TKR function had greater ability to improve, thereby increasing the likelihood of attaining the MCII, but decreasing the likelihood of attaining the PASS for post-TKR function because they needed greater improvement to achieve it. The opposite was true of subjects with better TKR function. The effect of baseline score on MCII attainment has been demonstrated previously $13,14,17,18$; however, our study is the first, to our knowledge, to examine the relationship between relative improvement and absolute functional status after

Table 2. Proportion of subjects by combination of post-total knee replacement (TKR) outcomes, and demographics of each group.

\begin{tabular}{|c|c|c|}
\hline & Attained PASS for TKR & Did Not Attain PASS for TKR \\
\hline Attained MCII & $41 \%$ & $3 \%$ \\
\hline \multirow{3}{*}{ Did not attain MCII } & ( $69 \%$ women, $92 \%$ white, $16 \%>75$ yrs old) & ( $100 \%$ women, $57 \%$ white, $0 \%>75$ yrs old) \\
\hline & $30 \%$ & $26 \%$ \\
\hline & ( $74 \%$ women, $85 \%$ white, $12 \%>75$ yrs old) & ( $73 \%$ women, $81 \%$ white, $20 \%>75$ yrs old) \\
\hline
\end{tabular}

PASS: Patient Acceptable Symptom State; MCII: minimal clinically important improvement.

Personal non-commercial use only. The Journal of Rheumatology Copyright (C) 2014. All rights reserved. 


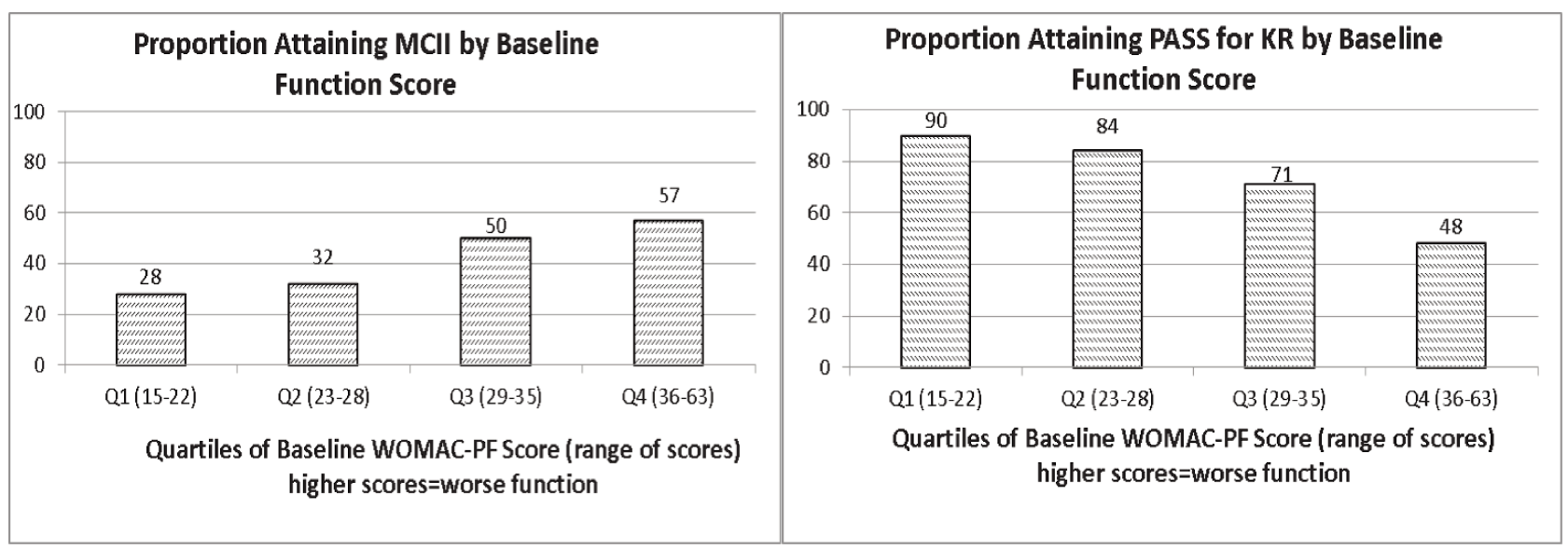

Figure 1. Proportion of subjects attaining each measure of post-total knee replacement function by baseline function score. MCII: minimal clinically important improvement; PASS: Patient Acceptable Symptom State; KR: knee replacement; WOMAC-PF: Western Ontario and McMaster Universities Osteoarthritis Index Physical Function subscale.

TKR. Our sensitivity analyses confirmed that the baseline score, rather than the time between pre-TKR assessment and surgery is more closely associated with attainment of a good outcome. Clinicians should consider baseline function when transmitting information on expected TKR outcomes to patients, and note that the improvement or state experienced after TKR may depend on how one defines it.

There are limitations to our study. The mean time to followup after TKR was 1.8 years. We used the MCII threshold previously published for those who are 2 years post-TKR ${ }^{6}$, and the PASS threshold from subjects 1 year following TKR ${ }^{12}$. We feel that this difference is unlikely to have affected our results, as data suggest that only minimal changes in function occur between 1 and 2 years post-TKR ${ }^{10,19}$. The values we used for the MCII and the PASS differed from published thresholds for patients with various rheumatic diseases who are taking nonsteroidal antiinflammatory therapy ${ }^{7}$. There is recent evidence suggesting that PASS values may be too low in rheumatic disease populations ${ }^{20}$. However, we used values determined specifically for TKR, and it is reasonable to expect that thresholds for pharmacologic versus surgical interventions would have different levels of acceptable response. We conducted a sensitivity analysis in which we used values at the upper and lower bounds of the $95 \%$ CI for each outcome (published in Escobar $2012^{12}$ and calculated from data in Escobar $2007^{6}$ ). This resulted in $40 \%$ to $51 \%$ of subjects meeting the MCII and $65 \%$ to $76 \%$ of subjects attaining the PASS. Both the $75 \%$ threshold and the mean for determining the MCII have been used in other studies ${ }^{6,7,21}$. If, as in the PASS, we had used the $75 \%$ threshold for MCII, then even fewer of our subjects would have met the MCII threshold and the discordance between PASS and MCII would have been greater.

In the main analysis, we did not exclude subjects whose pre-TKR function was at or above the PASS for TKR function because one of our aims was to assess the proportion with good absolute function after TKR, regardless of the degree of relative improvement. This may explain why $90 \%$ and $84 \%$ of the subjects in the best 2 quartiles of prereplacement function attained the PASS and why many fewer attained the MCII. Lastly, our analyses focused on a self-report measure of function and may not be similar to findings using performance-based measures of function.

If MCII in function is attained, an acceptable level of functioning after knee replacement is also likely to be attained. However, if MCII in function is not achieved, the likelihood of functioning at an acceptable level post-TKR depends on pre-TKR functional status. Further research is warranted on timing of surgery in relation to presurgical functional status and its effect on outcomes, as well as on understanding patient preferences regarding the importance of relative improvement in function versus absolute level of function. Insight into these concepts will be important in developing consensus regarding the optimal definition of successful functional outcomes post-TKR ${ }^{4}$, and ultimately would facilitate comparative effectiveness research for TKR.

\section{REFERENCES}

1. Kane R, Saleh KJ, Wilt TJ, Bershadsky B. The functional outcomes of total knee arthroplasty. J Bone Joint Surg Am 2005;87A:1719-24.

2. Singh JA, O'Byrne M, Harmsen S, Lewallen D. Predictors of moderate-severe functional limitation after primary Total Knee Arthroplasty (TKA): 4701 TKAs at 2-years and 2935 TKAs at 5-years. Osteoarthritis Cartilage 2007;18:515-21.

3. Kurtz S, Ong K, Lau E, Mowat F, Halpern M. Projections of primary and revision hip and knee arthroplasty in the United States from 2005 to 2030. J Bone Joint Surg Am 2007;89:780-5.

4. Losina E, Katz J. Total TKR: pursuit of the paramount result. Rheumatology 2012;51:1735-6.

5. Tubach F, Ravaud P, Baron G, Falissard B, Logeart I, Bellamy N, et al. Evaluation of clinically relevant states in patient reported 
outcomes in knee and hip osteoarthritis: the patient acceptable symptom state. Ann Rheum Dis 2005;64:34-7.

6. Escobar A, Quintana JM, Bilbao A, Arostegui I, Lafuente I, Vidaurreta I. Responsiveness and clinically important differences for the WOMAC and SF-36 after total knee replacement. Osteoarthritis Cartilage 2007;15:273-80.

7. Tubach F, Ravaud P, Martin-Mola E, Awada H, Bellamy N, Bombardier C, et al. Minimum clinically important improvement and patient acceptable symptom state in pain and function in rheumatoid arthritis, ankylosing spondylitis, chronic back pain, hand osteoarthritis, and hip and knee osteoarthritis: results from a prospective multinational study. Arthritis Care Res 2012; 64:1699-707.

8. Perrot S, Bertin P. "Feeling better" or "feeling well" in usual care of hip and knee osteoarthritis pain: Determination of cutoff points for patient acceptable symptom state (PASS) and minimal clinically important improvement (MCII) at rest and on movement in a national multicenter cohort study of 2414 patients with painful osteoarthritis. Pain 2013;154:248-56.

9. Felson DT, Niu J, Guermazi A, Roemer F, Aliabadi P, Clancy M, et al. Correlation of the development of knee pain with enlarging bone marrow lesions on magnetic resonance imaging. Arthritis Rheum 2007;56:2986-92.

10. Davis A, Perruccio AV, Ibrahim S, Hogg-Johnson S, Wong R, Streiner DL, et al. The trajectory of recovery and the inter-relationships of symptoms, activity and participation in the first year following total hip and TKR. Osteoarthritis Cartilage 2011;19:1413-21.

11. Bellamy N, Buchanan WW, Goldsmith CH, Campbell J, Stitt LW. Validation study of WOMAC: a health status instrument for measuring clinically important patient relevant outcomes to antirheumatic drug therapy in patients with osteoarthritis of the hip or knee. J Rheumatol 1988;15:1833-40.

12. Escobar A, Gonzalez M, Quintana JM, Vrotsou K, Bilbao A, Herrera-Espiñeira C, et al. Patient acceptable symptom state and OMERACT-OARSI set of responder criteria in joint replacement. Identification of cut-off values. Osteoarthritis Cartilage 2012; 20:87-92.
13. Hawker G, Wright J, Coyte P, Paul J, Dittus R, Croxford R, et al. Health-related quality of life after TKR. J Bone Joint Surg Am 1998;80:163-73.

14. Fortin PR, Penrod JR, Clarke AE, St-Pierre Y, Joseph L, Bélisle P, et al. Timing of total joint replacement affects clinical outcomes among patients with osteoarthritis of the hip or knee. Arthritis Rheum 2002;46:3327-30.

15. Felson DT, Niu J, Gross KD, Englund M, Sharma L, Cooke TD, et al. Valgus malalignment is a risk factor for lateral knee osteoarthritis incidence and progression: findings from the Multicenter Osteoarthritis Study and the Osteoarthritis Initiative. Arthritis Rheum 2013;65:355-62.

16. Tubach F, Dougados M, Falissard B, Baron G, Logeart I, Ravaud P. Feeling good rather than feeling better matters more to patients. Arthritis Care Res 2006;55:526-30.

17. Lingard EA, Katz JN, Wright EA, Sledge CB, and the Kinemax Outcomes Group. Predicting the outcome of total knee arthroplasty. J Bone Joint Surg Am 2004;86:2179-86.

18. Goldsmith $\mathrm{CH}$, Boers M, Bombardier C, Tugwell P. Criteria for clinically important changes in outcomes: development, scoring and evaluation of rheumatoid arthritis patient and trial profiles. OMERACT Committee. J Rheumatol 1993;20:561-5.

19. Leibs TR, Herzberg W, Roth-Krueger A, Ruther W, Hassenpflug J. Women recover faster than men after standard knee arthroplasty. Clin Orthop Relat Res 2011;469:2855-65.

20. Heiberg T, Kvien TK, Mowinckel P, Aletaha D, Smolen JS, Hagen $\mathrm{KB}$. Identification of disease activity and health status cut-off points for the symptom state acceptable to patients with rheumatoid arthritis. Ann Rheum Dis 2008;67:967-71.

21. Jaeschke R, Singer J, Guyatt GH. Measurement of health status. Ascertaining the minimal clinically important difference. Control Clin Trials 1989;10:407-15. 